\title{
Lesezeichen Nr. 5: Hol den Vorschlaghammer. Deutschsprachige Popmusilk - Romantiker und Antiromantiker
}

\author{
NADJA NIISSCHE \\ Salamanca
}

\begin{abstract}
Während in Deutschland emsthaft über Quoten für deutsche (oder deutschsprachige?) Popmusik im Radio diskutiert wird, kommt in Spanien kaum jemand auf die Idee, Popmusik in einer anderen als der eigenen Sprache zu hören. Spanische Deutschlerner werden sich über Songs mit deutschen Texten also vielleicht weniger wundern als das deutsche MTV-Publikum, obwohl das ja mittlerweile auch sehr auf Xavier Naidoo, Silbermond, Juli, Annette Louisan und all die anderen steht; von Altbewährten wie den Toten Hosen, Marius Müller-Westernhagen oder Nena ganz zu schweigen.

Da sich die Lesezeichen mit Literatur beschäftigen, haben wir für die folgenden Lektüre-(und Hör-) Empfehlungen Songs ausgewählt, die interessante Textarbeit ermöglichen, für die wir mit unseren Interpretationen Anregungen geben wollen. Dabei möchten wir - passend zum Heftthema - Lieder vorstellen, die sich im Spannungsfeld von Romantik und Antiromantik bewegen.
\end{abstract}

Paul van Dyk / Im Sommer 2004 Peter Heppner: $\quad$ machte ein Musik"Wir sind wir» video in Deutsch(2004) land Furore, das in suggestiver TextBild-Kombination markante Einschnitte der deutschen Nachkriegsgeschichte im Zeitraffer beschwor: «Wir sind wir» von Paul van Dyk und Peter Heppner.' Ausgangs- und Endpunkt der in diesem Video erzählten Geschichte markieren Bilder des Berliner Reichstages 1945 und heute, wobei das Schlussbild in raffinierter berblendung für den Bruchteil einer Sekunde noch einmal den Blick auf die Fassade in ihrem zerstörten Zustand freigibt und so den Wandel zwischen Kriegsende und heute in visu- ell beeindruckender Weise sichtbar macht. Dazwischen sieht man einen verwundeten Wehrmachtssoldaten, der durch die Trümmer Berlins humpelt, dumpf brummende Rosinenbomber, die Berlin mit Lebensmitteln während der Blockade 1948/49 versorgen, (teilweise farbige) Ausschnitte aus dem Berner WM-Finale von 1954, Bilder vom Mauerbau 1961, Ausschnitte aus einer westdeutschen Wochenschau, die das Wirtschaftswunder der fünfziger und sechziger Jahre dokumentieren, idyllische Vorgartenszenen aus jener Zeit (ebenfalls in Farbe) und schließlich Aufnahmen vom Mauerfall 1989. Seine Suggestivität zieht dieser Bilderbogen nicht zum wenigsten aus der Tatsache, dass der begleitende Erzähler immer ein und derselbe ist: Peter Heppner tritt in "Wir sind wir" als altersloser Beobachter auf, der, als Journalist mit Schreibmaschine und Handkamera ausgerüstet, im verwackelten Dogma-Stil das Schicksal der (West) Deutschen von 1945 bis heute Revue passieren lässt. Und was er uns zeigt, ist ganz ohne Zweifel eine Aufstiegs-, eine Erfolgsgeschichte, die freilich mit dem grüblerischen Refrain desLiedeskontrastiert: «Dochich frag, ich frag mich, wer wir sind, / «Und ich frag, ich frag mich, wo wir stehen.» Während die Bilder von «Wir sind wir» ein unzweideutiges "Auferstanden aus Ruinen" zum Ausdruck bringen, artikuliert der Text an ver-

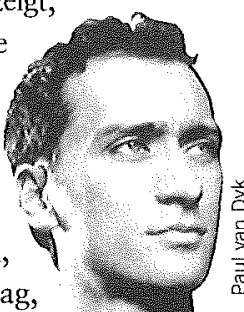

schiedenen Stellen grundsätzliche Selbstzweifel:

Doch bleiben viele Fenster leer, Für viele gab es keine Wiederkehr. Und über das, was grad noch war, Spricht man heute lieber gar nicht [mehr.

(Strophe 2)

Auferstanden aus Ruinen dachten wir, Wir hätten einen Traum vollbracht. 40 Jahre zogen wir an einem Strang. Aus Asche haben wir Gold gemacht.

Jetzt ist mal wieder alles anders Und was vorher war, ist heute nichts [mehr wert.

Jetzt können wir haben was wir [wollen, Aber wollten wir nicht eigentlich viel [mehr?

(Strophen 5 und 6)

Doch was hier auf den ersten Blick als selbstkritische Bestandsaufnahme des westdeutschen Wohlstands- und Wirtschaftswunders, sogar - siehe Strophe 2 - als Kritik an der Verdrängung von Vergangenheit verstanden werden könnte, ist in Wahrheit trotziges Beharren auf der Fähigkeit des "Wir", alle Schwierigkeiten zu meistern:

Wir sind wir! Wir stehen thier! Aufgeteilt, besiegt und [doch, Schließlich leben wir ja [noch.

Wir sind wir! Wir stehen [hier! 
Das kanns noch nicht gewesen sein.

Keine Zeit zùm Traurigsein.

Wir sind wir! Wir stehn' hier!

Wir sind wir!

(Strophen 3 und 4)

Wir sind wir! Wir stehen hier!

Wieder Eins in einem Land,

Superreich und abgebrannt.

Wir sind wir! Wir stehen hier! So schnell kriegt man uns nicht klein, Keine Zeit zum bitter sein.

Wir sind wir! Wir stehn' hier!

Wir sind wir!

\section{Wir sind Wir!}

Aufgeteilt, besiegt und doch, Schließlich gibt es uns ja immer noch

Wir sind wir!

Und wir werden's überstehen,

Denn das Leben muss ja weitergehen.

\section{Wir sind wir!}

Das ist doch nur ein schlechter Lauf. So schnell geben wir doch jetzt nicht [auf.

\section{(Strophen 7 - 11)}

Als Sinnbild dieser Fähigkeit fungiert in «Wir sind wir» die deutsche Fußballnationalmannschaft, die 1954 die Weltmeisterschaft gewann und damit zur kollektiven Projektionsfläche jener "Wir sind wieder wer»Mentalität wurde, die - gespeist von außenpolitischen, wirtschaftlichen und eben nicht zuletzt auch sportlichen Erfolgen - das Selbstbewusstsein der Westdeutschen in den fünfziger Jahren entscheidend bestimmte. Die Refrainformel "Wir sind wir» ist leicht als eine Anspielung auf dieses «Wir sind wieder wer» zu entschlüsseln. Indem van Dyk und Heppner den 2004 ohnehin mit groBer Aufmerksamkeit bedachten historischen WM-Sieg so demonstrativ in den Mittelpunkt rücken, suggerieren sie, dass die komplexe, ebenso ökonomische wie moralische Krise der Gegenwart - «superreich und abgebrannt» heißt es in bewusst paradoxer Formulierung - nur in gemeinschaftlicher Kraftanstrengung, also auf dem Wege einer wie auch immer zu denkenden kollektiven Mobilisierung zu bewältigen ist. Das Setzen aufs Kollektiv als Reaktion auf Krisen ist freilich nicht neu, sie hat ihre Vorläufer, die bis zur Wende vom 18. zum 19. Jahrhundert zurückreichen. Die damalige Rückbesinnung der deutschen Romantik auf Nation, Geschichte und Religion war der Versuch, den als krisenhaft wahrgenommenen Auflösungserscheinungen der sich um 1800 rasant beschleunigenden Modernisierung etwas dem Wandel Entzogenes entgegenzusetzen, der freilich von vorneherein Gefahr lief, ins dumpf Konservative, gar Reaktionäre umzuschlagen ${ }^{2}$. Bewusst oder unbewusst knüpfen van Dyk und Heppner mit ihrer tautologischen Leerformel des "Wir sind wir" an diese Tradition an, ihre Ästhetik lässt sich als eine Aktualisierung solcher konservativen Romantik deuten. Der Anschluss an diese Tradition erklärt auch zu einem guten Teil, warum «Wir sind wir» den Zuhörer" und Zuschauer zu bewegen vermag, allerdings nicht ohne dabei ein unbestimmtes Unbehagen auszulösen. Georg Diez beschreibt den Sound von "Wir sind wir" in der Frankfurter Allgemeinen Sonntagszeitung in ebenso zutreffender wie verräterischer Formulierung als «ein über das Brüllen, über das Wollen, über den Trotz gelegter elektronischer Klangfluß, der einen fast rauschhaft mitziehen will» - man ahnt in diesem "will" die Angst des Kritikers vor dem vielleicht etwas peinlichen Eingeständnis, dass dieses Ziel auch durchaus erreicht wird. Und es verwundert andererseits nicht, dass das Video in bestimmten Teilen des Feuilletons sehr gut ankam. So zeigte sich etwa Susanne Leinemann in der Welt tief beeindruckt und nahm van Dyk / Heppner gegen Kritik in Schutz :

«Und während man vom Pop-Pathos überwältigt auf dem Sofa sitzt, nimmt man verwundert dieses neue Gefühl wahr, durch ein Musikvideo von Deutschland und seiner Nachkriegsgeschichte ergriffen zu sein. So oft gab es das nicht, ein gutes Lied über diese Nation - ohne Ironie, ohne Abwertung, ohne Distanz. Im Gegenteil, voller Anteilnahme. Und mit jeder neuen Woche steigt die Spannung, ob Heppner und van Dyk unbeschadet 
Sechziger, das erscheint der Kritik gefährlich. [...] Am Ende bleibt "Wir sind wir» ein Popstück - seine Halbwertzeit ist kurz. Aber der Streit um Lied und Video ist symptomatisch für eine Generation, die sich in den Neunzigern unter dem Dreigestirn Ironie, Spaßkultur, Nostalgie fand und nun auseinander läuft. Einige laufen weiter fort. Einige drehen sich um und erkennen ihr Land. Was ist schlecht daran?

Auf diese - wie uns scheint: gewollt naive Frage ließe sich mit Georg Diez antworten, dass "Wir sind wir» den «deutsche[n] Opferschmerz, nicht de[n] Stolz der Befreiten" zum Ausdruck bringt, und dies mit einer Musik unterlegt, «die [...] alle Zweifel fort[flutet]».' Statt historischer Aufklärung also vernebelnde Inszenierung, statt kritischer Selbstreflexion Wiederbelebung problematischer Mythen - verantwortlicher Umgang mit der deutschen Nachkriegsgeschichte sieht anders aus. Trotzdem oder vielleicht sogar gerade deshalb eignet sich «Wir sind wir» für den DaF-Unterricht hervorragend, lässt das Video doch niemanden unberührt und bietet zugleich zahlreiche Anknüpfungspunkte zu vertiefender, kritischer Diskussion.

\section{Herbert Vonder «deutschen Grönemeyer: Innerlichkeit» zum «Mensch» (2002) Themenbereich, dem sich die Pop- musik traditionell eher widmet: dem der privaten Gefüh-}

le. Herbert Grönemeyers «Mensch» (aufdem gleichnamigen Album von 2002) hat keinerlei «Plot», keine äußere Handlung; sondern es geht darum, eine (momentane, so das erste Wort des Textes) Stimmung einzufangen:

Momentan ist richtig, momentan ist gut nichts ist wirklich wichtig

nach der Ebbe kommt die Flut

am Strand des Lebens

ohne Grund, ohne Verstand

ist nichts vergebens

[...]

alles auf dem Weg

und es ist Sonnenzeit

unbeschwert und frei

(Zeile 1-11)

Es fallen zunächst eine Reihe positiver Formulierungen auf, umgangssprachliche oder neutrale: "nichts ist wirklich wichtig", "alles auf dem Weg», "es ist okay» (Zeile 8), und auch poetisch verfremdete, die sich aber leicht entschlüsseln lassen: «Sonnenzeit», «am Strand des Lebens", "Das Firmament hat geöffnet, / wolkenlos und ozeanblau" (Zeile 18f.). Was für eine Stimmung es genau ist, die hier vermittelt wird, lässt sich aber gar nicht so leicht feststellen: «Telefon, Gas, Elektrik / unbezahlt, und das geht auch» (Zeile 20f.) - das erinnert an das romantische Lebensgefühl des Eichendorffschen Taugenichts, der auf Gott und sein Glück vertraut und sich um die materiellen Dinge des Lebens keine Sorgen macht. "Nach der Ebbe kommt die Flut» (Zeile 3) klingt nach einem ebenfalls romantischen Vertrauen auf die unabänderlichen Zyklen der Natur; die das Leben bestimmen und menschliches Glück und Unglück relativieren.

Auf den zweiten Blick wird diese ausgeglichene, wenn nicht positive Weltsicht jedoch brüchig. «Nach der Ebbe kommt die Flut» lässt sich, in dieser Reihenfolge, zwar übersetzen als «nach dem Schlechten kommt wieder Gutes»; umgekehrt folgt aber auf die Flut auch wieder die Ebbe; auf jedes Glück das nächste Unglück.

Derart aufmerksam geworden, findet man in den meisten anderen vordergründig lebensbejahenden Textzeilen ähnliche Doppeldeutigkeiten. So gleich in der ersten Zeile: «Momentan ist richtig, momentan ist gut»-was immer hier 'richtig' und 'gut' ist, ist jedenfalls nicht von Dauer; die Telefonrechnung nicht zu bezahlen könnte man auch als Zeichen fortgeschrittener Depression deuten; und das "es ist okay", das vierStrophen einleitet, ist ein Satz, den man im Deutschen eigentlich nur als Trostfloskel ver- wenden kann, also gerade in Situationen, in denen nichts okay ist.

Nun überrascht auch nicht weiter; das dieselben vier Strophen enden mit "du fehlst». Hier wird eine Lücke bezeichnet, ein Mangel oder Verlust; die um diese Liikke herum aufgebotene Romantik offenbart sich - strukturell ähnlich wie bei van Dyk/ Heppner - als Reaktion auf eine Krise. Ein Hinweis auf den medienbekannten biographischen Hintergrund dieses und anderer Songs des Albums - den Tod von Grönemeyers Frau im Jahre 1998 - drängt sich an dieser Stelle geradezu auf, zumal er dem Text vielleicht eine Aura von Ehrlichkeit und tatsächlich erlebtem Leid verleiht, die andere Popsongtexte nicht für sich beanspruchen können. Allerdings darf man dabei nicht übersehen, dass uns Lesern / Hörern nur der Popsong zugänglich ist, und nicht der Mensch dahinter; die überlieferte Biographie ist ein Teil des Textes, und nicht etwa umgekehrt. Mit anderen Worten: Den Text auf eine biographische Interpretation festzulegen, reduziert ihn auf nur eine von stets mehreren möglichen Lesarten; im Unterrichtsgespräch ist es daher erfahrungsgemäß ergiebiger, den biographischen Aspekt des Textes gar nicht zu erwähnen, um der

Vorstellung einer «richtigen» Deutung des Textes vorzubeugen.

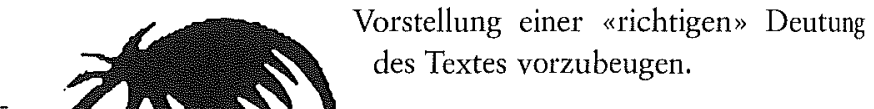

Wir sind Helden, Eine Identifi«Denkmal» kation desly(2003) rischen Ichs mit dem Sänger bzw. der Sängerin bietet sich auch bei diesem Song an; genauer gesagt handelt es sich hier um ein «lyrisches Wir», das auffällig mit dem "Wir» im Namen der Band übereinstimmt. Vom Denkmal, um das es im Songtext geht, kommt man per Assoziation ohnehin leicht zu den namengebenden Helden.

Außer diesem an sich schon romantisch aufgeladenen Begriff «Held» lassen sich leicht eine ganze Reihe weiterer romantischer Versatzstücke finden: Gleich in der ersten Zeile wird ein Fenster 


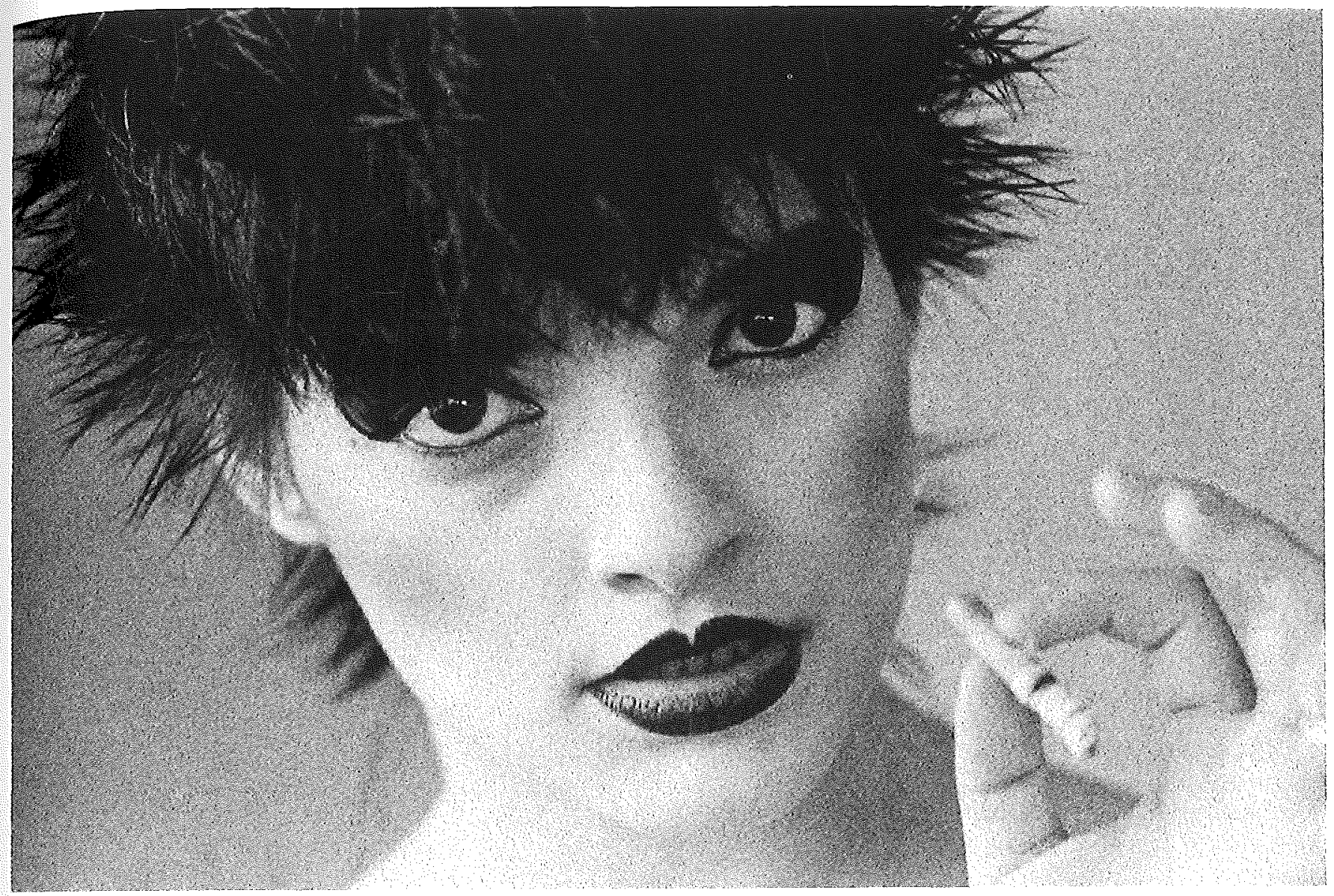

Nina Hagen

erwähnt, das für den Blick in die Ferne, die -ffnung zu einem anderen Raum, den «bergang in eine andere (bessere, erträumte) Welt stehen kann. "Komm her zu mir» (ebenfalls Zeile 1) könnte in einem Film oder einem anderen Song eine Liebesszene einleiten. Ein «Aldi» (Zeile 3) lässt vielleicht an Armutsromantik denken; die «Blumen und Girlanden halb zerknüllt» (Zeile 6) womöglich an Clemens Brentanos «Ponce de Leon». Von den «schlechtesten Sprayer[n] dieser Stadt» (Zeile 13) ist die Rede, bei denen das romantische Motiv des Underdog anklingt. Sogar ein literaturhistorisch noch weit ehrwürdigeres Motiv wird zitiert: «Es wird bald hell und wir haben nicht ewig Zeit» (Zeile 18) - das skizziert die Ausgangssituation des hochmittelalterlichen Tagelieds, in dem sich zwei Liebende bei Tagesanbruch voneinander verabschieden müssen. Auf dem Umweg über die Mittelalterbegeisterung der Romantiker lässt sich auch dies an die romantische Motivreihe anschließen.

All diese Korrespondenzen sind zugegebenermaßen vage; und sie werden auch nur als Vorgabe für die jeweils sogleich erfolgen- de Ironisierung gebraucht: In der zuletzt zitierten Zeile wird das «du» zur Eile gedrängt, weil das «ich» Maßnahmen gegen das titelgebende Denkmal ergreifen will, das «uns» gebaut worden ist, also wahrscheinlich den Helden, nach denen sich die Gruppe explizit performativ benennt - sie heißen eben nicht «Helden» oder «Die Helden», sondern "Wir sind Helden", machen also den Akt des Benennens zum Bandnamen und damit hinfällig. Denn Helden, die sich selbst so nennen, sind entweder Angeber oder ironisch. Diese hier wollen jedenfalls kein Denkmal gesetzt bekommen: «Wenn uns jetzt hier wer erwischt sind wir für immer vereint / in Beton und Seligkeit» (Zeile 19). Das Denkmal, zumal wenn aus schnödem Beton gefertigt, symbolisiert den Tod, wenn auch vielleicht weniger biologischen als den geistigen / menschlichen / emotionalen: «Da steht in goldener Schrift wir sollen in Ewigkeit ruhen». Mit dem Vorschlaghammer, nach dem im Refrain gerufen wird, soll das Denkmal zertrümmert werden, denn «jeder Vollidiot weiß / dass das [nämlich ein Denkmal] die Liebe versaut» (Zeile 11f.).
Hier zeigt sich nun allerdings, dass die Helden mit der rabiaten Vorgehensweise gegen unbestellte Denkmale nicht etwa ihre Anonymität wahren, möglichen Heldenkult unterbinden oder eigener Eitelkeit vorbauen, sondern - die Liebe retten wollen. Wenn das kein romantisches Anliegen ist!

\section{Nina Hagen: Mit dem Ende ih- Du hast rer Liebe hingegen den Farbfilm droht ganz offen vergessen (1974) Nina Hagen in ih- remStück «Duhast denFarbfilm verges- sen» aus dem Jah-}

re 1974, den wir denn auch als ein bemerkenswertes Beispiel für musikalische Antiromantik vorstellen wollen. Die Szenerie, die hier vom lyrischen Ich gezeichnet wird, ist vordergründig durchaus romantisch: «Hoch stand der Sanddorn am Strand von Hiddensee» (Strophe 1); von "Sand" und "Himmelblau» (ebd.) ist im weiteren die Rede. Diesem Ferien- und Urlaubsidyll kontrastiert indes sehr komisch die Wut, mit der hier dieses Ich - Nina - auf die Tatsache 
reagiert, dass ihr Freund - Michael - den «Farbfilm» vergessen hat: «Tu das noch einmal, Micha, und ich geh!» (ebd.).

Du hast den Farbfilm vergessen, mein [Michael

Nun glaubt uns kein Mensch, wie schön's [hier war

Du hast den Farbfilm vergessen, bei [meiner Seel'

Alles blau und weiß und grün und [später nicht mehr wahr

Du hast den Farbfilm vergessen, bei [meiner Seel'

Alles blau und weiß und grün und [später nicht mehr wahr

(Refrain)

Nicht der Urlaub mit dem Geliebten ist offenbar das Entscheidende, sondern die Fähigkeit, anschließend mit entsprechenden Urlaubsdokumenten auftrumpfen zu können: «Nun glaubt uns kein Mensch, wie schön's hier war."

Das heute durchaus aus künstlerischen Gründen geschätzte Schwarzweiß kann angesichts der Konvertierung des Farbfilms zum erschwinglichen - wenn auch offenbar noch nicht überall leicht erhältlichen - Massenprodukt nicht mehr konkurrieren. Diese antiromantische Emphase transportiert freilich auch ein gehöriges Stück politischer - dabei durchaus ambivalenter Ironie: Die Farbfotografie ermöglicht es dem Reisenden, den Nachweis zu führen, dass die Welt keineswegs so grau ist wie

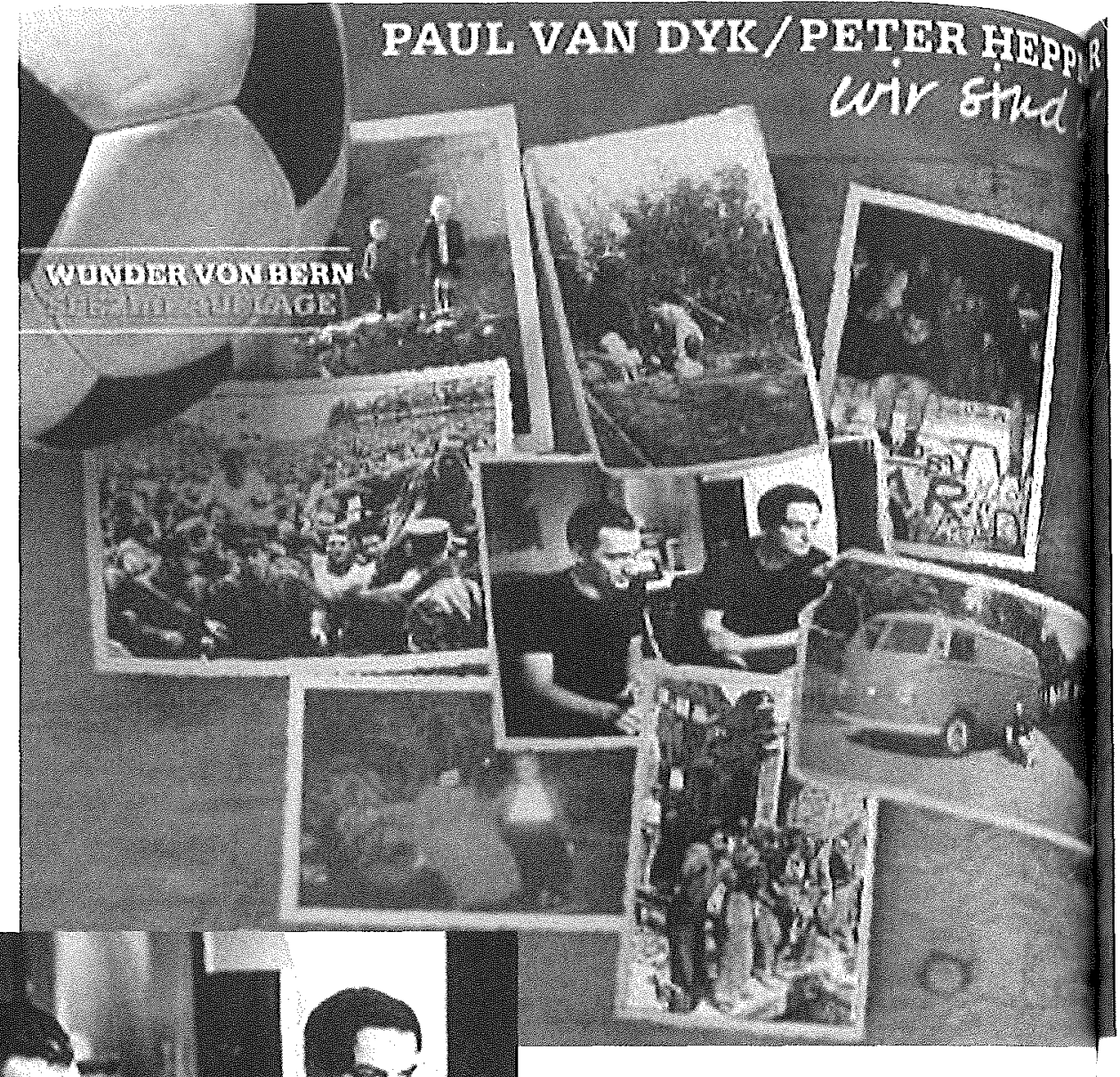

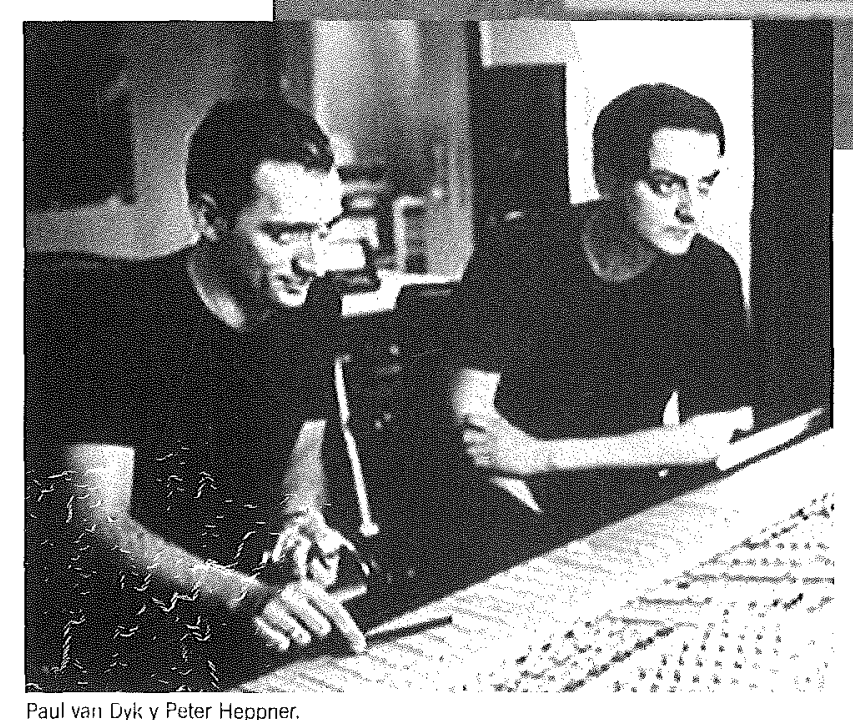

Paul van Dyk y Peter Heppner. sie dem an die Tristesse des DDR-Alltags gewöhnten Daheimgebliebenen vorkommen mochte. Mehr noch: Aus dem frechschmollenden, kindlich trotzigen Tonfall Ninas spricht eine Freude an der Welt, an den Dingen, am eigenen Körper und eine Lust an der Zurschaustellung desselben, die schon für sich genommen provokativ sind und den antiromantischen Gestus des Liedes unterstreichen:

Ich im Bikini und ich am FKK

Ich frech im Mini, Landschaft ist auch da - ja
Aber wie schrecklich, die Tränen kullern heiß

Landschaft und Nina und alles nur schwarzweiß

Micha, mein Micha, und alles tut so weh 'Tu das noch einmal, Micha, und ich geh'? (Strophe 2)

Hier wird in einem geradezu burlesken Auftritt im Kirmessound der Lust am Diesseits das Wort geredet, dass es jedem $\mathrm{An}^{-}$ tiromantiker eine Freude sein muss. Die eigentliche Pointe liegt dabei darin, dass dadurch ganz nebenbei die DDR als das
Land profiliert wird, in dem solche unverkrampfte Lebensfreude - "ich am FKK" ihre genuine Heimat hat. Subtil konstruiert der «Farbfilm»-Song damit so etwas wie eine eigenständige, und zwar: antiromantische Identität der DDR, die in dem alten sozialistischen Versprechen eines "Himmelreichs auf Erden" (Heine, Wimtermärchen) seine Wurzeln hat. Nicht zufällig endet Leander Haußmanns 1998 entstandener Film Sonnenallee mit Nina Hagens «Du hast den Farbfilm vergessen", setzt doch «Sonnenallee» als die erste grö Bere filmische Darstellung der DDR einen - ebenfalls trotzigen - Kontrapunkt zur bis heute üblichen DDR-Verdammung. Und zeigt doch zugleich, dass das antiromanti sche Versprechen, als das sich die DDR selbst begriff, mit zu ihrem eigenen Untergang beigetragen hat, weil der Abstand zwischen Realität und Versprechen zunehmend unerträglicher wurde. Doch dass die Geschichte so ausgehen würde, hätte man freilich schon 1974 ahnen können, als $\mathrm{Ni}$ na Hagen mit böse aufstampfenden nack tem Fuß ihr Recht auf Farbe einforderte. 


\section{Diskographie}

- Wir sind Helden, «Denkmal», in: Die Reklamation, 2003, Label: Labels (EMI). - Herbert Grönemeyer, «Mensch», in; Mensch, 2002, Groenland (EMT). - Paul van Dyk \& Peter Heppner, "Wir sind wird", in: Wir sind wir, 2004, Urban (Universal).

- Nina Hagen, «Du hast den Farbfilm vergessen», in: Sonnenallee (Soundtrack), 2002, Eastwest (Warner Music).

Die Songtexte sind außer in den Booklets der CDs (noch) im Internet zu finden; allerdings nicht auf den offiziellen Homepages der Musiker (auf der Helden-Homepage http://www.wirsindhelden.com/gibt es immerhin die Texte der aktuellen CD «Von hier an blind"), sondern auf Fan-Homepages, die sich über eine Suchmaschine leicht recherchieren lassen.

\section{Anmerkungen}

1. Webseite: http://artists.universalmusic.de/wirsindwir/. Dort kann man sich das Stück auch anhören.

2. So ambivalent wie die Modernisierung, auf die sie reagierte, war daher auch die Romantik selbst. Einer der bedeutendsten Kritiker der Romantik, Heinrich Heine, kann sich paradoxerweise daher zugleich als ihr «letzter und abgedankter Fabelkönig» verstehen (Brief an Varnhagen vom 3. Januar 1846, zit. nach Gerhard Höhn: Heine-Handbuch. Zeit, Person, Werk, Stuttgart: Metzler 1987, 257). Im «Wintermärchen» führt dieser romantische Antiromantiker am Beispiel des preußischen Militärs in ironischer Weise die zur politischen Macht gekommene reaktionäre Romantik vor und prophezeit ihren baldigen Untergang:

Nicht übel gefiel mir das neue Kostüm Der Reuter, das muß ich loben, Besonders die Pickelhaube, den Helm Mit der stählernen Spitze nach oben.

Das ist so rittertümlich und mahnt An der Vorzeit holde Romantik, An die Burgfrau Johanna von Montfaucon, An den Freiherrn Fouqué, Uhland, Tieck.

Das mahnt an das Mittelalter so schön, An Edelknechte und Knappen, Die in dem Herzen getragen die Treu' Und auf dem Hintern ein Wappen.

Das mahnt an Kreuzzug und Turnei, An Minne und frommes Dienen, An die ungedruckte Glaubenszeit, Wo noch keine Zeitung erschienen.

Ja, ja, der Helm gefällt mir, er zeugt Vom allerhöchsten Witze!

Ein königlicher Einfall war's!

Es fehlt nicht die Pointe, die Spitze!

Nur fürcht ich, wenn ein Gewitter entsteht,

Zieht leicht so eine Spitze

Herab auf euer romantisches Haupt

Des Himmels modernste Blitze! -
Heinrich Heine: Deutschland. Ein

Wintermärchen, in: Digitale Bibliothek Sonderband: Meisterwerke deutscher Dichter und Denker, S. 18391-18392 (vgl. Heine-WuB Bd. 1, S. 441).

3. Susanne Leinemann: «Unter Verdacht. Wolfsheim singen von deutschen Mythen» $<$ http:/www.welt,de/data/2004/08 /27/324390.html> (2.3.2005).

4. Georg Diez: «Neuer deutscher Pop. Böhse Enkelz», in: FAS 22.8.04. Im Internet unter der folgenden, etwas sperrigen Adresse zu finden: http://www.faz.net/s/RubE219BC35AB30 426197C224F193F54B1B/Doc EC18F3 AEF76D7493A97A3FE166342193F AT pl Ecommon Scontent.html (25,4.2005).

5. Noch schärfer die Kritik von Marvin Alster: «Dabei gehen van Dyk und Heppner mit «Wir sind Wir» über die bloße Verdrehung des Täter-OpferVerhältnisses - der 2. Weltkrieg und der Holocaust finden im Lied keine Erwähnung, sehr zentral aber die Schäden der Bombardierung Berlins - hinaus, wenn sie vor dem Hintergrund des "Wirtschaftswunders» bewusst mehrdeutig texten: «Jetzt können wir haben was wir wollen / Aber wollten wir nicht eigentlich viel mehr?" Mit anderen Worten: Alles Materielle war wieder zu kaufen, Volkswagen, Persil und Nylonstrümpfe, aber eigentlich strebten die Deutschen nach Höherem. Spätestens ab dieser Zeile spielen Heppner und van Dyk mit einer Ambivalenz, die ihnen immer eine Hintertür offen hält, aber dennoch ihre Intention des völkischen Revanchismus offenbart. Die Deutschen wollten tatsächlich viel mehr; «Lebensraum» und völkische Reinheit, einschließlich der Judenvernichtung, " Marvin Alster: «Wir sind wir. Das popkulturelle Deutschland fährt völkisches Geschütz auf", in: Phase 2 $<$ http://phase2.nadir.org/index.htm> (2.3.2005). 